\title{
Energy and frequency measurements on the Twente Raman free-electron laser
}

\author{
P.J.M. van der Slot \\ Nederlands Centrum voor Laser Research B.V., P.O. Box 2662, 7500 CR Enschede, The Netherlands
}

\author{
W.J. Witteman \\ University of Twente, Department of Applied Physics, P.O. Box 217, 7500 AE Enschede, The Netherlands
}

Results of energy and frequency measurements on the Twente Raman FEL are presented and discussed. A highest energy of $230 \mathrm{~mJ}$ was measured using a joule meter. The spectrum was determined using waveguide cutoff filters. These measurements generally showed a broad spectrum containing two or more peaks. Far from magneto-resonance this indicates that the radiation field grows at the upper as well as at the lower interaction of the slow space-charge wave with the the electromagnetic waveguide modes. Near magneto-resonance, the analysis of the spectra shows the presence of the cyclotron instability together with the FEL instability. The two instabilities are resonant with different waveguide modes. The latter was confirmed by a measurement of the radial profile of the emitted radiation for a single setting of the guide and undulator field.

\section{Introduction}

The first experiments done with the Twente Raman FEL have been focused on the determination of the total emitted energy with the corresponding spectra. The FEL has been described before [1] so the main parameters are only briefly mentioned here. The FEL uses a $500 \mathrm{kV}$ electron pulse of $100 \mathrm{~ns}$ duration with a current increasing from $\simeq 600 \mathrm{~A}$ at the beginning to $\simeq 900 \mathrm{~A}$ at the end of the pulse. The voltage ripple is less than $2 \%$ apart from a little overshoot at the beginning of the pulse. The helical undulator has a period of $3 \mathrm{~cm}$ and a tapered entrance over six periods. The waveguide has an inner radius of $8 \mathrm{~mm}$.

To obtain the total emitted energy and the corresponding spectra, two types of experiments were performed. In the first a specially designed joule meter was mounted directly at the end of the waveguide. The joule meter [2] uses a pyramid, made out of a silicon wafer, as an absorber. Four NTC thermistors (Philips type 232262622104 ), connected in series, are used to measure the temperature change. The joule meter was calibrated using a heater mounted on the absorber as well as using a known if pulse [3]. Both types of calibration were in excellent agreement and resulted in a sensitivity of $8.9 \pm 0.4 \mathrm{k} \Omega / \mathrm{J}$ (at $T=293 \mathrm{~K}$ ). Some of the power falling on the joule meter will be reflected. This has experimentally [3] been determined to be less than $-15 \mathrm{~dB}$ for the fundamental $\mathrm{TE}_{11}$ mode. The detection threshold was estimated to be $4 \mathrm{~mJ}$, corresponding to an average power of $40 \mathrm{~kW}$ for a rf pulse of $100 \mathrm{~ns}$ duration. For typical experimental conditions the heat leakage was $\simeq 60 \mu \mathrm{J} / \mathrm{s}$ and the equilibrium temperature was reached in less than $15 \mathrm{~s}$ after absorption of the radiation pulse.

For the second type an outcouple horn has been used to emit the radiation into free space and small sectoral horns are used to feed waveguide cutoff filters. The vacuum window placed in the horn will result in a small amount of reflected power which is less than $-20 \mathrm{~dB}$ over the whole $\mathrm{K}_{\mathrm{a}}$ band (26.5-40 $\mathrm{GHz}$ ) [3]. Crystal detectors (Hewlett Packard type HP R422C) operating in the $\mathbb{K}_{\mathrm{a}}$ band are used to measure the filtered signals. Free-space propagation is used to attenuate the rf signal to levels below the damage threshold of the crystal detectors. This has been determined experimentally to be $10^{4}$ [3] for a circular polarised $\mathrm{TE}_{11}$ wave. The filters have a cutoff wavelength ranging from 6 to $12 \mathrm{~mm}$ with $1 \mathrm{~mm}$ increment. In the experiment two filters with successive cutoff wavelengths are placed aside each other and in line with the laser at a distance of $1.8 \mathrm{~m}$ from the outcouple horn. The signal of each detector is integrated and the difference between the two integrals gives the energy emitted in the wavelength interval between the two cutoff wavelengths. A third detector subtends a small angle at the outcouple horn and uses a $\mathrm{K}_{\mathrm{a}}$-band waveguide as a receiver. This detector is used to monitor and compensate shot-to-shot variations.

Before presenting results of the measurements it must be mentioned that the experimental conditions for the frequency and energy measurements are not 
identical. For the energy measurement an open waveguide is used to radiate the energy into the absorber whereas for the frequency measurements the outcouple horn with vacuum window (having different if characteristics) is used. In both cases an oscillator is formed. For the energy measurement the round-trip power loss is $98 \pm 1 \%$ for frequencies in the $\mathrm{K}_{\mathrm{a}}$ band whereas for the frequency measurements it is $99.5 \pm$ $0.4 \%$. Although the difference seems small care must be taken using results from one type of experiment in the other.

\section{Energy measurements}

The total energy in the laser pulse has been investigated as a function of the guide field $B_{0}$ for three different values of the undulator field. The results are shown in figs. 1, 2 and 3. In these figures each open circle represent a single measurement whereas the filled circles indicate several measurements yielding the same energy in the pulse. The error in each measurement is $4 \mathrm{~mJ}$. For all three figures the average energy of the electrons is within the interval $1.966<\gamma$ $<$ 1.993. In each case the guide field $B_{0}$ is varied between $\approx 0.3 \mathrm{~T}$ and $1.75 \mathrm{~T}$. The lower boundary is set by the requirement of stable beam propagation through the waveguide whereas the upper boundary is determined by the power supply of the magnet. The orbital stability boundaries for type I $\left(B_{\mathrm{cr}, \mathrm{I}}\right)$ and type II $\left(B_{\mathrm{cr}, \mathrm{II}}\right)$ steady state orbits in a realistic undulator field [4] are shown for convenience only.

A highest energy of $\simeq 230 \mathrm{~mJ}{ }^{\# 1}$ has been measured for an undulator field amplitude $B_{\mathrm{u}}=0.19 \mathrm{~T}$ for

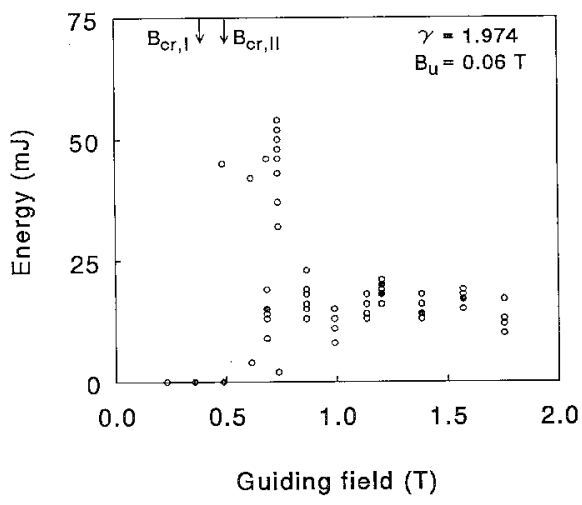

Fig. 1. Total energy within the laser pulse as a function of the guide field $B_{0}$ for $B_{\mathrm{u}}=0.06 \mathrm{~T}$. The orbital stability boundaries for the type $\mathrm{I}\left(B_{\mathrm{cr}, \mathrm{I}}\right)$ and type II $\left(B_{\mathrm{cr}, \mathrm{II}}\right)$ steady state orbits in a realistic undulator field are shown for convenience only. The electron energy $\gamma=1.974 \pm 0.008$. Each circle represents a single measurement, a filled circle indicates several measurements yielding the same result.

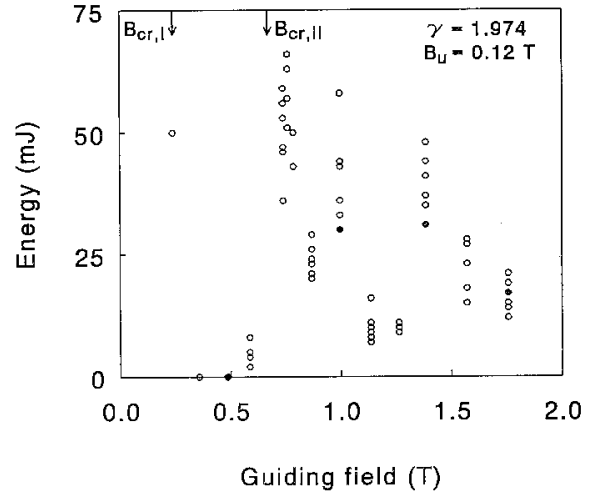

Fig. 2. As fig. 1 but for an undulator field $B_{\mathrm{u}}=0.12 \mathrm{~T}$.

the guide field $B_{0}=0.36 \mathrm{~T}$ as well as $B_{0}=1.38 \mathrm{~T}$ (see fig. 3). Taking the FWHM of the radiation pulse equal to the electron pulse duration the maximum average power is $2.3 \mathrm{MW}$. However a few remarks about this average power can be made. The energy in the $K_{a}$ band is also measured by integrating the signal of a crystal detector positioned at $1.80 \mathrm{~m}$ from and in line with the outcouple horn. The free-space attenuation has been determined experimentally for the $\mathrm{TE}_{11}$ mode (see above) since the nonlinear code ARACHNE [5] predicts that the FEL instability is only resonant with this mode. We observed that for almost all of the magnetic field combinations investigated the energy measured with the joule meter is higher than the energy obtained from the crystal detector data. This leads to the conclusion that the emitted spectrum is wider than the $\mathrm{K}_{\mathrm{a}}$ frequency band, higher order modes are present in the laser pulse or a combination of both.

From the measured spectra (see next section) it can be deduced that in the neighbourhood of magneto-resonance $\left(\Omega_{\mathrm{o}} / c k_{\mathrm{u}}=\beta_{z}\right.$, where $\Omega_{\mathrm{o}}=e B /(\gamma m)$ is the relativistic cyclotron frequency, $k_{\mathbf{u}}=2 \pi / \gamma_{\mathrm{u}}$ is the undulator wavenumber and $\beta_{z}=v_{z} / c$ is the normalised axial velocity of the electrons) higher order modes are present in the laser pulse. This indicates that the cyclotron instability is present as well. Far from magneto-resonance no contributions from the cyclotron instability are expected and found in the measured spectra. Here the difference in total energy and the energy emitted in the $K_{a}$ band indicates that the laser pulse contains frequencies below the $K_{a}$ band $(\leq 26.5$ $\mathrm{GHz}$. These frequencies will probably correspond to the lower intersection of the slow space-charge wave on the electron beam $\left(\omega=\left(k+k_{u}\right) c \beta_{z}-\omega_{\mathrm{p}} / \gamma_{z} ; k\right.$ is the axial wavenumber of the radiation mode and $\omega_{\mathrm{p}}$ is the relativistic plasma frequency) with the $T E_{11}$ wave-

\footnotetext{
\#1 The total kinetic energy present in the electron beam is $\simeq 45 \mathrm{~J}$ for $B_{0} \geq 0.7 \mathrm{~T}$.
} 


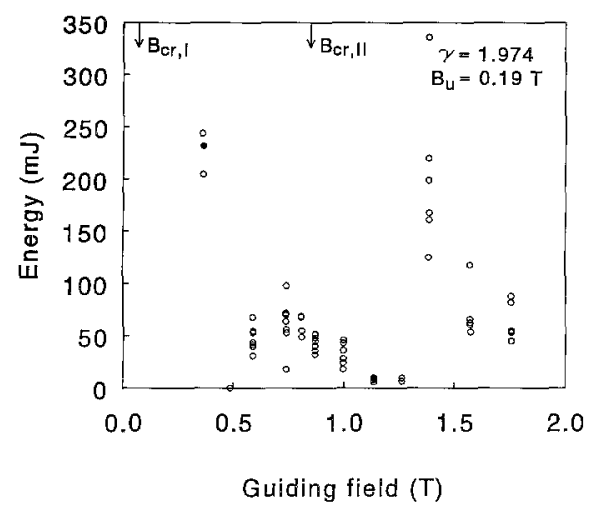

Fig. 3. As fig. 1 but for an undulator field $B_{\mathbf{u}}=0.19 \mathrm{~T}$.

guide mode $\left(\omega^{2}=c^{2} k^{2}+\omega_{c}^{2}, \omega_{c}\right.$ being the cutoff frequency).

From the raw crystal-detector signals it can be seen that the FWHM of the if pulse may be up to a factor of 5 smaller than the FWHM of the electron pulse. From figs. 1 to 3 it follows that the spread in the measurements increase with increasing total energy. This may be related to the high gain present in the system and the fact that the laser starts from noise.

\section{Frequency measurements}

The waveguide cutoff filters used in the experiment approximately limit the measurements to the $\mathrm{K}_{\mathrm{a}}$ frequency band $(26.5-40 \mathrm{GHz})$. The measurements are thus focused on the upper intersection of the dispersion relations of the electromagnetic and slow spacecharge waves. The spectral content of the radiation pulse has been determined for several combinations of $B_{0}$ and $B_{\mathrm{u}}$. Two typical results are shown in figs. 4 and 5 for $B_{0}=0.74$ and $B_{0}=1.00$ respectively. In both cases the undulator field amplitude $B_{u}=0.06 \mathrm{~T}$ and electron energy $\gamma=1.974$.

The nonlinear code ARACHNE [5] shows a growth of the FEL instability at wavelengths $\lambda \simeq 27 \mathrm{~mm}$ and $\lambda \simeq 11 \mathrm{~mm}$ for the parameters of fig. 4 . The latter agrees rather well with the observed wavelength of $\simeq 11.5 \mathrm{~mm}$. The lower frequency is below the detection capability. ARACHNE allows for multiple waveguide modes but only the $\mathrm{TE}_{11}$ mode seems to be resonant with the FEL instability at these frequencies. The other observed peak at $\simeq 7.5 \mathrm{~mm}$ must be attributed to the cyclotron instability for the following reasons. First the guide field is close to magneto-resonance. So it can be expected that the electron orbit contains a considerable Larmor contribution. Furthermore, using the selection rules for the cyclotron maser instability [6,7], one finds a cyclotron instability resonant with the $\mathrm{TM}_{01}$

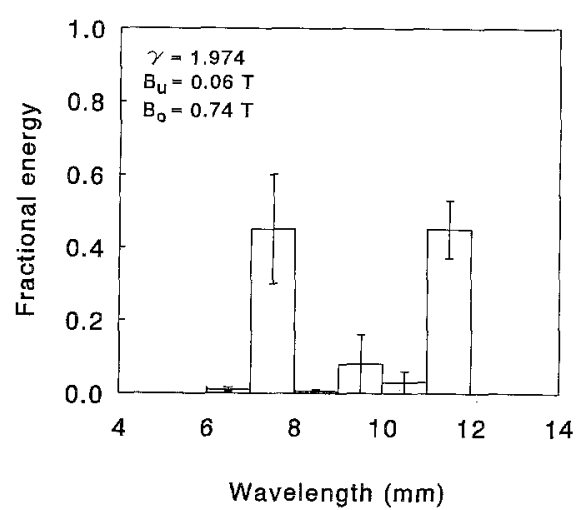

Fig. 4. Spectrum of the radiation pulse within the $\mathrm{K}_{\mathrm{a}}$ band. The electron energy is $\gamma=1.974 \pm 0.008$. Each bar represents the fractional energy emitted in the wavelength interval given by the width of the bar. The magnetic fields are $B_{\mathrm{u}}=0.06 \mathrm{~T}$ and $B_{0}=0.74 \mathrm{~T}$.

mode at a wavelength of $7.1 \mathrm{~mm}$. This value agrees very well with the observed peak at $7.5 \mathrm{~mm}$ (see fig. 4). From fig. 4 it follows that the $\mathrm{TE}_{11}$ (FEL) and $\mathrm{TM}_{01}$ (cyclotron) modes contain an equal amount of energy. Taking the radial patterns of the two modes into account, this leads to the conclusion that the cyclotron instability is the dominant instability. This is confirmed by measuring the radial radiation profile at $1.90 \mathrm{~m}$ distance from the outcouple horn. Two crystal detectors were used, one was positioned on axis and the other at a varying angle from the axis and fixed azimuthal angle. The result is shown in fig. 6 where the peak intensity (in arbitrary units) is shown as a function of the distance to the axis. The distribution shown here is consistent with a superposition of a $\mathrm{TE}_{11}$ and a $\mathrm{TM}_{01}$ mode, the latter being dominant. Note that the distance between the antenna (i.e. outcouple horn) and receiver is large enough to be at the boundary of the far-field region of the antenna $\left(2 D^{2} / \lambda \leq 1.83 \mathrm{~m}\right.$ for $\lambda \geq 7 \mathrm{~mm}$, where $D$ is the diameter of the antenna).

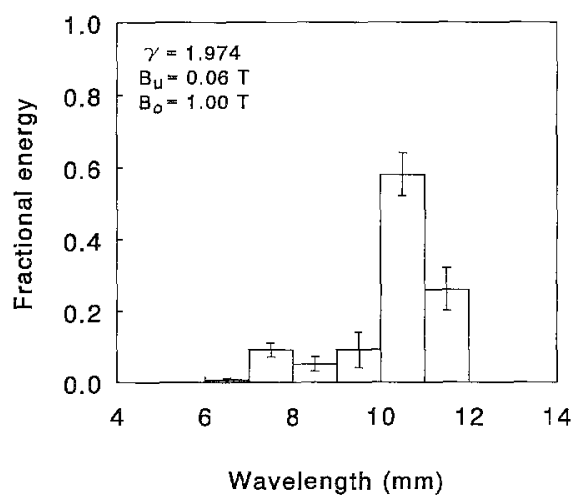

Fig. 5. As fig. 4 but the magnetic fields are $B_{\mathrm{u}}=0.06 \mathrm{~T}$ and $B_{0}=1.00 \mathrm{~T}$. 


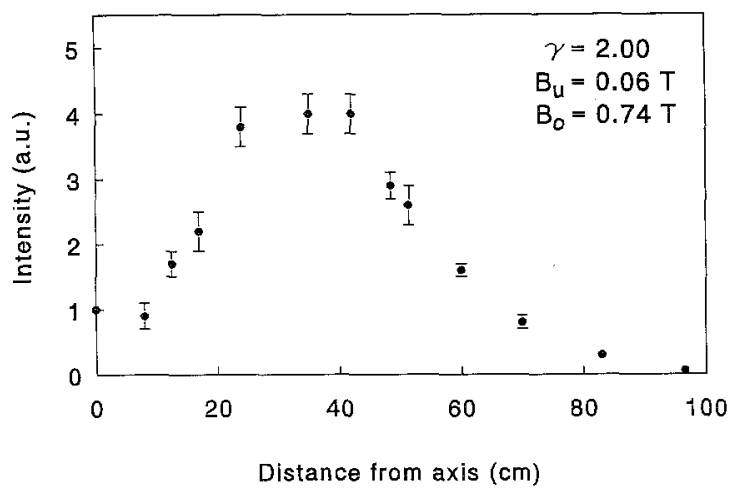

Fig. 6. Radial distribution of the intensity for $B_{\mathrm{u}}=0.06 \mathrm{~T}$ and $B_{0}=0.74 \mathrm{~T}$. The distance between outcouple horn and receiving horn is $1.90 \mathrm{~m}$.

The ARACHNE code shows maximum efficiency at $\lambda \simeq 8.8 \mathrm{~mm}$ for the parameters corresponding to fig. 5 $\left(B_{0}=1.00 \mathrm{~T}, B_{\mathrm{u}}=0.06 \mathrm{~T}\right)$. This wavelength is smaller than the measured peak at $\simeq 10.5 \mathrm{~mm}$. ARACHNE seems to predict too small wavelengths compared to linear theory and experimental results whenever the intersections with the waveguide mode are too far separated from each other (as is the case for fig. 5) [5]. This is attributed to an underestimate of the plasma frequency in the space-charge modes. For the case of near-grazing intersections the underestimate is relatively small (as is the case for fig. 4). The possibility of cyclotron instability has been investigated too and a resonance is found with the $\mathrm{TE}_{01}$ waveguide mode at a wavelength $\lambda=10.6 \mathrm{~mm}$. However, it is expected that the injection of electrons onto steady-state orbits will be good. Therefore the cyclotron instability will be weak and the peak found at $\simeq 10.5 \mathrm{~mm}$ must be attributed to the FEL instability.

\section{Conclusions}

The first measurements on the Twente Raman FEL indicate that, for the parameters investigated, a laser pulse is generated with a broad spectrum. Near magneto-resonance this is due to the simultaneous pres- ence of FEL and cyclotron instability resonant with different waveguide modes at different frequencies. Further away from magneto-resonance only the FEL instability is found showing gain on the upper as well as the lower intersections of the slow space-charge wave dispersion curve with the electromagnetic waveguide dispersion curve of the $\mathrm{TE}_{11}$ mode. This follows from the observation that the the total energy in the laser pulse (as measured with the joule meter) is usually higher than the energy emitted in the $K_{a}$ frequency band (calculated from the crystal detector data). The energy measurements resulted in a maximum energy of $\simeq 230 \mathrm{~mJ}$ per pulse for the strongest undulator field investigated $\left(B_{\mathrm{u}}=0.19 \mathrm{~T}\right)$. The frequency measurements (limited to the $26.5-40 \mathrm{GHz}$ band) show separated peaks with a FWHM of $\simeq 1 \mathrm{~mm}$ and a wavelength varying between 7 and $12 \mathrm{~mm}$.

\section{Acknowledgements}

The authors would like to thank C. Penman for the useful discussions and P.J.S. Teunisse from Hollandse Signaalapparaten B.V., The Netherlands and A.F.M. Bouman for their assistance with the if diagnostics.

\section{References}

[1] P.J.M. van der Slot, C. Penman and W.J. Witteman, Proc. 12th Int. FEL Conf., Paris, France, 1990, Nucl. Instr. and Meth. A304 (1991) 268.

[2] R.H. Jackson, PhD Thesis, North Carolina State University, USA (1984); and P.J.M. van der Slot, PhD Thesis, University of Twente, The Netherlands (1992).

[3] Measurements were performed at the Development department microwave components and antennas of Hollandse Signaalapparaten B.V. Hengelo, The Netherlands.

[4] P. Diamond, Phys. Rev. A23 (1981) 2537.

[5] A.K. Ganguly and H.P. Freund, Phys. Fluids 31 (1990) 387.

[6] T.C. Marshall, in: Laser Handbook, vol 6, Free Electron Lasers, eds. W.B. Colson, C. Pellegrini and A. Renieri (North-Holland, 1990).

[7] H.P. Freund, S. Johnston and P. Sprangle, IEEE J. Quantum Electron. QE-19 (1983) 322. 